\title{
Impact of Clinical Decision Support on Azithromycin Prescribing in Primary Care Clinics
}

\author{
Alexandria May, PharmD, BCPS ${ }^{\top}$, Allison Hester, PharmD', \\ Kristi Quairoli, PharmD, BCACP, CDCES ${ }^{7}$, Jordan R. Wong, PharmD, BCPS ${ }^{7}$, and \\ Sheetal Kandiah, $\mathrm{MD}^{2}$
}

'Department of Pharmacy, Grady Health System, Atlanta, GA, USA; ²Division of Infectious Disease, Emory University School of Medicine, Atlanta, GA, USA.

BACKGROUND: Inappropriate use of antibiotics in the outpatient setting is a common problem, yet literature evaluating best practices for stewardship interventions in this setting is sparse.

OBJECTIVE: To evaluate the impact of clinical decision support (CDS) order panels for azithromycin prescribing on the percentage of inappropriate azithromycin prescriptions in primary care clinics.

DESIGN: Single-center, retrospective analysis of azithromycin prescribing within nine primary care clinics. Pre-intervention and post-intervention data included azithromycin prescriptions from November 2016 to April 2017 and February 2019 to July 2019, respectively. Key exclusion criteria included prescriptions for the treatment of a sexually transmitted infection or for prophylaxis against Mycobacterium avium complex.

INTERVENTION: The azithromycin CDS panel was created to provide point-of-care information on appropriate use of azithromycin along with recommended alternatives based on indications. CDS panels were implemented on January 10, 2019.

MAIN MEASURES: The primary composite outcome was the change in the percentage of inappropriate azithromycin prescribing before and after implementation of CDS panels. The composite outcome included prescriptions with inappropriate indications for azithromycin, unnecessary prescriptions, inappropriate treatment durations, and/or inappropriate dose.

KEY RESULTS: There were 306 and 263 prescriptions for azithromycin prescriptions included for analysis in the pre- and post-intervention periods, respectively. Inappropriate prescriptions decreased by $12.6 \%$ from the pre- to post-intervention period ( $81.4 \%$ vs. $68.8 \% ; P<0.001)$. In both the pre- and post-intervention period, bronchitis and unspecified upper respiratory tract infections (URI) were the two most common indications where azithromycin was prescribed inappropriately.

CONCLUSIONS: Implementation of CDS order panels resulted in a reduction in inappropriate azithromycin prescribing. However, additional improvement in

Prior Presentations An earlier version of this manuscript was presented as a poster at the American Society of Health-System Pharmacists Midyear Meeting in Las Vegas, Nevada, in 2019.

Received July 1, 2020

Accepted December 22, 2020

Published online February 25, 2021 azithromycin prescribing is needed especially for the indications of bronchitis and unspecified URI.

KEY WORDS: clinical decision support; antibiotic stewardship; ambulatory; primary care.

J Gen Intern Med 36(8):2267-73

DOI: $10.1007 / \mathrm{s} 11606-020-06546-\mathrm{y}$

(c) Society of General Internal Medicine 2021

\section{BACKGROUND}

Improving antibiotic prescribing is critical to preventing drug resistance and adverse effects as well as minimizing excessive use of healthcare resources and associated costs. ${ }^{1}$ Recently, there has been a growing focus on antibiotic stewardship in primary care practices as $>60 \%$ of antibiotic expenditures are associated with the outpatient setting. ${ }^{1}$ In 2016, the Centers for Disease Control and Prevention released the Core Elements of Outpatient Antibiotic Stewardship, which include commitment, action for policy and practice, tracking and reporting, and education and expertise. ${ }^{1}$ Although the Core Elements lay the foundation for outpatient antibiotic stewardship programs (ASP), literature evaluating the effectiveness of antibiotic stewardship (AS) interventions in this setting are sparse.

A national study estimated that $30 \%$ of outpatient antibiotic prescribing was inappropriate according to professional society guidelines. ${ }^{2}$ However, this is likely an underestimation of inappropriate prescribing. ${ }^{3}$ For instance, a study evaluating inappropriate outpatient prescribing in primary clinics within a Veterans Affairs Health Care System found that $76 \%$ of prescriptions were considered inappropriate when agent selection and duration were also considered. ${ }^{4}$ Specifically, this study found that azithromycin was one of the top offending prescriptions, with $78.4 \%$ of outpatient azithromycin prescriptions considered inappropriate according to prescribing guidelines. ${ }^{4}$ Likewise, another study found that azithromycin was the most common agent prescribed inappropriately at their institution, with $55 \%$ of prescriptions considered unnecessary and $32 \%$ of prescriptions considered to be for an indication in which azithromycin is not indicated. ${ }^{5}$ Data from these studies supports outpatient AS interventions targeted to specific highrisk antibiotic agents such as azithromycin. 
Clinical decision support (CDS) tools have been used to reduce inappropriate prescribing of antibiotics for acute respiratory tract infections in the ambulatory care setting. For example, a study conducted at 33 primary care practice sites within an integrated health system found a reduction in inappropriate antibiotic prescribing for acute bronchitis after implementation of either print (from 80.0 to $68.3 \%$ ) or computerized (from 74.0 to $60.7 \%$ ) CDS in conjunction with provider and patient education. ${ }^{6}$ Likewise, another study evaluated three behavioral interventions - suggested alternatives, peer comparison, or accountable justification - on the rate of inappropriate prescribing for acute respiratory tract infections among 47 primary care practices. $^{7}$ All three interventions resulted in lower rates of inappropriate prescribing, with inappropriate antibiotic prescribing decreasing by $16.0 \%, 16.3 \%$, and $18.1 \%$ for suggested alternatives, peer comparison, and accountable justification, respectively.

Azithromycin is a broad-spectrum antibiotic that is commonly overprescribed for conditions where antibiotics are not recommended, such as acute bronchitis, non-specific upper respiratory infections (URI), or viral pharyngitis. ${ }^{1}$ At our institution, CDS was developed in the form of an order panel within the electronic medical record (EMR) to evaluate its effect on the percentage of inappropriate azithromycin prescribing within primary care clinics.

\section{METHODS}

\section{Study Design}

This study was a single-center, retrospective analysis of a quality improvement project of nine primary care clinics within Grady Health System (GHS). The study was approved by the Emory University investigational review board. Four academic primary care clinics are attached to the main hospital and consist of attending and resident physicians. Five nonacademic, primary care clinics are off-site of the main hospital and consist of attending physicians and advance practice providers (APPs). Outpatient azithromycin prescriptions were identified from the EMR (EPIC; Verona, WI).

\section{Inclusion/Exclusion Criteria}

The pre-intervention and post-intervention data included azithromycin prescriptions from the primary care clinics from November 1, 2016, to April 30, 2017, and February 1, 2019, to July 31, 2019, respectively. Patients were excluded if they were younger than 18 years of age, pregnant or incarcerated, diagnosed with multiple infections, failed previous treatment, or had more than two antibiotic allergies. Additionally, prescriptions for azithromycin for treatment of a sexually transmitted infection or for prophylaxis or treatment against $\mathrm{Myco}$ bacterium avium complex (MAC) or febrile neutropenia were excluded.

\section{CDS Panels}

The pre-intervention data was reported at an ambulatory care quality meeting that included the primary care clinic medical directors on December 12, 2019. The CDS panels were demonstrated for the medical directors who were then responsible for disseminating this information to the providers in their respective clinics. Before CDS panel implementation, clinicians could directly order azithromycin outpatient prescriptions from computer order entry without restriction or guidance for use.

The azithromycin CDS panel was implemented on January 10, 2019. The CDS panels were created in collaboration with the pharmacy information technology team associated with the EMR. The purpose of the azithromycin order panel was to provide real-time education and guidance on appropriate use of azithromycin at the time of order entry along with suggested alternatives based on indication. Standard ordering was replaced with the CDS order panel for all outpatient prescriptions for azithromycin. The clinician was required to select an option within the order panel where guidance and alternative options were also provided. However, free-standing ordering of azithromycin was still permitted within the order panel; therefore, the panel was non-restrictive. Ordering azithromycin oral suspension did not direct the clinician to the azithromycin order panel.

The azithromycin order panel contained brief text information for appropriate use of azithromycin (Fig. 1). References to institutional guidelines for URIs or FDA warnings associated with azithromycin were provided via hyperlink. The azithromycin order panel provided the clinician with only one selection based on indication. Each selection provided a default dose and duration. The panel indications included bronchitis, sinusitis, community-acquired pneumonia, sexually transmitted infections, and MAC prophylaxis. Additional azithromycin orders within the panel offered the $250 \mathrm{mg}$ and $600 \mathrm{mg}$ tablet size without defaulting prescribing information, to allow prescribing outside of common indications.

Additional order panels for bronchitis and sinusitis were created and linked within the azithromycin panel. The bronchitis and sinusitis order panels, respectively, provided medication options for symptom relief (Figs. 2 and 3). Within either the bronchitis or sinusitis order panel, the clinician could select as many medications as deemed appropriate. Both bronchitis and sinusitis order panel medications were based on national recommendations for symptom control that was individualized based on local practice and formulary. ${ }^{8-10}$

In addition, either bronchitis or sinusitis panels could be ordered, independent of the azithromycin panel, by searching for the panel name or key words associated with indications.

\section{Outcomes}

The primary composite outcome of this study was the change in the percent of inappropriate azithromycin prescribing before and after implementation of the CDS panels in the EMR. The 
Alternative Selection

Alternative Required

You selected:

azithromycin (ZITHROMAX) $250 \mathrm{mg}$ tablet: Take 2 tablets on day 1; Take 1 tablet on days 2-5. Disp-6 tablet, R-0, Normal

Details

- asthma and COPD exacerbations DO NOT require antibiotics

- do not use azithromycin for SSTIs, UTIs, sinusitis

- caution with use for patients with concurrent QTc prolonging medications

References

- YRRi Diagnostic Criteria c

- FDA Warning on FQs - mental health and hypoglycemia $c$

- FDA Warning on FQs - risk > benefit related to indications $C$

- Community-acquired Pneumonia (CAP) Guidelines

- FDA Warning on FQs - aortic dissection/aneurysm $c$

\begin{tabular}{|c|c|}
\hline Alternatives & \\
\hline Alternative & Details \\
\hline Bronchitis Treatment & albuterol HFA (VENTOLIN) 108 (90 Base) MCG/ACT inhaler, guaifenes... \\
\hline Sinusitis Treatment & This suggestion contains a panel. Review the orders before signing. \\
\hline Pneumonia 1 st line - azithromycin $500 \mathrm{mg} \times 1$, then $250 \mathrm{mg}$ daily $\times 4$ days & Disp-6 tablet, R-0, Normal \\
\hline Pneumonia 2 nd line - doxycycline $100 \mathrm{mg}$ PO BID $\times 5$ days & Disp-10 capsule, R-0, Normal \\
\hline Azithromycin (Z-pak) $500 \mathrm{mg} \times 1$ then $250 \mathrm{mg}$ daily $\times 4$ days & Disp-6 tablet, R-0, Normal \\
\hline GC/Chlamydia azithromycin $1 \mathrm{~g} \mathrm{PO} \times 1$ dose & Disp-2 tablet, R-0, Normal \\
\hline MAC prophylaxis $1200 \mathrm{mg} \mathrm{PO}$ once a week & Normal \\
\hline azithromycin (ZITHROMAX) $250 \mathrm{mg}$ tablet & Normal \\
\hline azithromycin (ZITHROMAX) $600 \mathrm{mg}$ tablet & Normal \\
\hline
\end{tabular}

Figure 1 Azithromycin clinical decision support panel for computer order entry.

- Please educate/counsel patient on the following:

- Bronchitis is a self-limiting disease - may take up to 6 weeks for complete resolution

- Commonly due to viruses

- Antibiotics DO NOT have benefit in treating viruses

- Giving antibiotics may cause harm including: diarrhea, rashes, severe diarrhea (C. difficile infection), anaphylaxis and future antibiotic resistance (future infection has resistance to antibiotics)

URI Diagnostic Criteria

$\square$ albuterol HFA (VENTOLIN) 108 (90 Base) MCG/ACT inhaler Disp-1 Inhaler, R-0, Normal

$\square$ guaifenesin-dextromethorphan (ROBITUSSIN DM) 100-10 MG/5ML syrup - Do NOT exceed 7 days of use Normal

$\square$ benzonatate (TESSALON) 100 mg capsule, Do not exceed 7 days of use Normal

$\square$ cetirizine (ZYRTEC) $10 \mathrm{mg}$ tablet Normal

$\square$ loratadine (CLARITIN) $10 \mathrm{mg}$ tablet Normal 


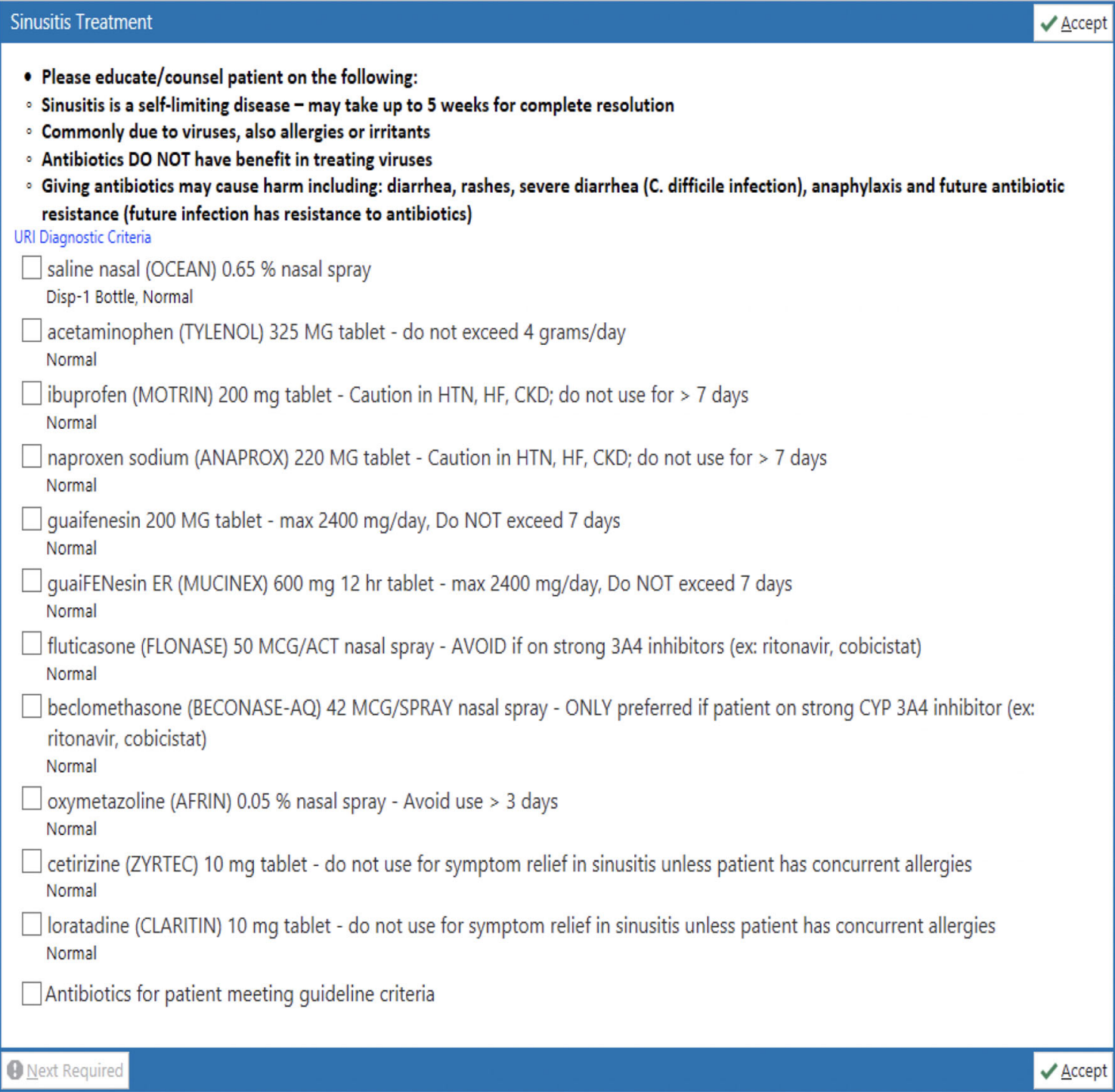

Figure 3 Sinusitis clinical decision support panel.

composite outcome included azithromycin prescriptions with an inappropriate indication, unnecessary prescription, excessive or insufficient treatment duration, and inappropriate dose. Inappropriate indication was defined as a prescription for a diagnosis in which azithromycin is not recommended. Unnecessary prescription was defined as a prescription for a diagnosis with no indication for antibiotics. Inappropriate treatment duration was defined as a prescription with an excessive or insufficient duration according to guidelines. Inappropriate dose was defined as a dose that deviated from the guideline-recommended dosage regimen for the particular condition being treated. Appropriateness of prescription was based on the institution's outpatient antibiotic guidelines, or national guidelines when institutional guidelines did not exist. ${ }^{8-10}$ Secondary outcomes included change in the percent of individual components of the primary outcome, patients requiring additional antibiotics within 30 days, and return visits to a GHS clinic or the emergency department (ED) within 30 days for azithromycinrelated adverse effects. Inappropriate prescribing between type of provider and type of clinic were evaluated.

\section{Statistics}

Primary and secondary outcome analyses were performed using chi-squared or Fisher's exact test, and a $P$ value of $<$ 0.05 was considered significant. Analysis was performed using IBM SPSS Statistics, Version 24.0 (2016; Armonk, New York).

\section{RESULTS}

There were 306 and 263 prescriptions for azithromycin included for analysis in the pre- and post-intervention periods, respectively (Fig. 4). Baseline characteristics associated with azithromycin prescribing are shown in Table 1. Consistent with pre-intervention data, prescriptions for azithromycin were most commonly written by attending physicians $(74.5 \%)$ in the post-intervention period. Likewise, inappropriate prescriptions most commonly originated in the nonacademic clinic setting (76.0\%) post-intervention. The most 


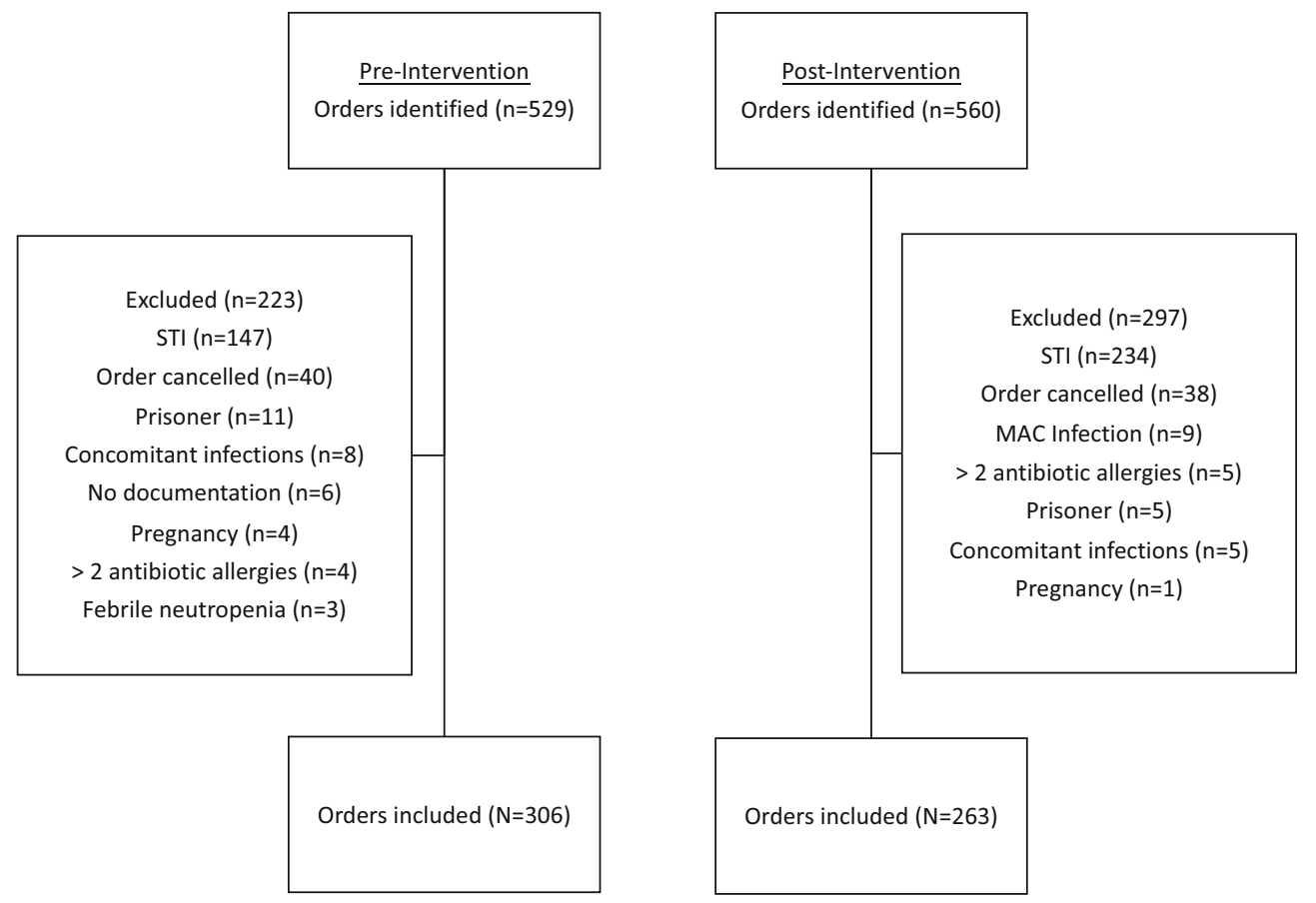

Figure 4 Flow diagram of inclusion and exclusions.

common indications for azithromycin were bronchitis or unspecified URI. The median duration of therapy was 5 days in both the pre- and post-intervention groups (Table 2).

Table 1 Baseline Characteristics Associated with Azithromycin Prescriptions

\begin{tabular}{|c|c|c|}
\hline Characteristic & $\begin{array}{l}\text { Pre-intervention } \\
(N=306)\end{array}$ & $\begin{array}{l}\text { Post-intervention } \\
(N=263)\end{array}$ \\
\hline Age (years), mean (SD) & $57.0(12.0)$ & $48.0(8.5)$ \\
\hline Female, $n(\%)$ & $223(72.9)$ & $182(69.2)$ \\
\hline \multicolumn{3}{|l|}{ Race, $n(\%)$} \\
\hline Black & $235(76.8)$ & $174(66.2)$ \\
\hline White & $30(9.8)$ & $31(11.8)$ \\
\hline Hispanic & $21(6.9)$ & $32(12.1)$ \\
\hline Other & $20(6.5)$ & $26(9.9)$ \\
\hline $\begin{array}{l}\text { Duration of therapy (days), } \\
\text { median (IQR) }\end{array}$ & $5(5.5)$ & $5(5.5)$ \\
\hline \multicolumn{3}{|l|}{ Provider, $n(\%)$} \\
\hline Attending physician & $222(72.5)$ & $196(74.5)$ \\
\hline Resident physician & $66(21.6)$ & $40(15.2)$ \\
\hline Advanced practice & $18(5.9)$ & $27(10.3)$ \\
\hline provider & & \\
\hline \multicolumn{3}{|l|}{ Clinic type, $n(\%)$} \\
\hline Non-academic & $218(71.2)$ & $200(76.0)$ \\
\hline Academic & $88(28.8)$ & $63(24.0)$ \\
\hline \multicolumn{3}{|c|}{ Indication, inappropriate/total prescriptions (\% inappropriate) } \\
\hline Bronchitis & $75 / 77(97.4)$ & $69 / 69(100)$ \\
\hline URI, unspecified & $108 / 110(98.2)$ & $61 / 62(98.4)$ \\
\hline Pneumonia & $0 / 28(0)$ & $1 / 37(2.7)$ \\
\hline COPD exacerbation & $11 / 28(39.3)$ & $15 / 32(46.9)$ \\
\hline Sinusitis & $37 / 38(97.4)$ & 17/17 (100) \\
\hline COPD, chronic & $0 / 5(0)$ & $0 / 10(0)$ \\
\hline Asthma exacerbation & $12 / 13(92.3)$ & $5 / 5(100)$ \\
\hline Otitis media & $0 / 2(0)$ & $1 / 5(20)$ \\
\hline Pharyngitis & $5 / 5(100)$ & 4/4 (100) \\
\hline Bronchiolitis & $0 / 3(0)$ & $0 / 3(0)$ \\
\hline Lymphadenopathy & $0 / 0(0)$ & $3 / 3(100)$ \\
\hline Traveler's diarrhea & $0 / 0(0)$ & $0 / 3(0)$ \\
\hline Other & $3 / 3(100)$ & $5 / 11(45.5)$ \\
\hline
\end{tabular}

URI, upper respiratory tract infection; COPD, chronic obstructive pulmonary disorder
The primary composite outcome of inappropriate azithromycin prescriptions decreased by $12.6 \%$ from the pre-

Table 2 Results of the Primary and Secondary Outcomes

\begin{tabular}{|c|c|c|c|}
\hline Outcome & $\begin{array}{l}\text { Pre- } \\
\text { intervention } \\
(N=306)\end{array}$ & $\begin{array}{l}\text { Post- } \\
\text { intervention } \\
(N=\mathbf{2 6 3})\end{array}$ & $\begin{array}{l}P \\
\text { value }\end{array}$ \\
\hline $\begin{array}{l}\text { Composite outcome for } \\
\text { inappropriate } \\
\text { prescription*, } n(\%)\end{array}$ & $249(81.4)$ & $181(68.8)$ & $\begin{array}{l}< \\
0.001\end{array}$ \\
\hline $\begin{array}{l}\text { Inappropriate indication, } \\
n(\%)\end{array}$ & $214(69.7)$ & $161(61.2)$ & 0.04 \\
\hline $\begin{array}{l}\text { Unnecessary } \\
\text { prescription, } n(\%)\end{array}$ & $208(67.8)$ & 147 (55.9) & 0.004 \\
\hline $\begin{array}{l}\text { Inappropriate duration, } \\
n(\%)\end{array}$ & $10(3.3)$ & $16(6.1)$ & 0.11 \\
\hline $\begin{array}{l}\text { Inappropriate dose, } \\
n(\%)\end{array}$ & $3(1.0)$ & $10(3.8)$ & 0.05 \\
\hline $\begin{array}{l}\text { Additional antibiotics } \\
\text { within } 30 \text { days, } n(\%)\end{array}$ & $6(1.9)$ & $5(1.9)$ & 0.96 \\
\hline Adverse events within 30 & $2(<0.1)$ & $0(0)$ & 0.51 \\
\hline
\end{tabular}

days ${ }^{\dagger+}, n(\%)$

Inappropriate prescription by provider type, inappropriate/total prescriptions (\% inappropriate)

Attending physician $\quad 189 / 222 \quad 153 / 196(78.1)-0.06$

$\begin{array}{llll} & (85.1) & & \\ \text { Resident physician } & 44 / 66(66.7) & 18 / 40(45.0) & 0.03\end{array}$

Advanced practice $\quad 16 / 18(88.9) \quad 10 / 27(37.0)<$

provider 0.001

Inappropriate prescription by clinic type, inappropriate/total prescriptions (\% inappropriate)

\begin{tabular}{llll} 
Non-academic & $190 / 218$ & $152 / 200(76.0)$ & 0.003 \\
Academic & $(87.2)$ & & \\
& $59 / 88(67.1)$ & $29 / 63(46.0)$ & 0.01 \\
\hline
\end{tabular}

*Primary composite outcome was percentage of azithromycin prescriptions classified by at least one of the following: inappropriate indication, unnecessary prescription, inappropriate duration, or inappropriate dose. Inappropriate indication = prescriptions for diagnoses in which azithromycin is not recommended; unnecessary prescription = diagnoses with no indication for antibiotics

tPatient-reported allergic reaction $(n=1)$ and disturbance in INR $(n=1)$ 
to post-intervention period (81.4\% vs. $68.8 \% ; P<0.001)$. There was an $8.7 \%$ decrease in prescriptions with inappropriate indications $(69.7 \%$ vs. $61.2 \% ; P=0.04)$ and $11.9 \%$ decrease in unnecessary prescriptions $(67.8 \%$ vs. $55.9 \% ; P=$ $0.004)$ from the pre- to post-intervention period. Although the numbers were small, in the post-intervention period, there was a slight increase in prescriptions with inappropriate durations (3.3\% vs. $6.1 \% ; P=0.11)$ and inappropriate doses $(1.0 \%$ vs. $3.8 \% P=0.05)$. In both the pre- and post-intervention period, $1.9 \%$ of patients required additional antibiotics within 30 days $(P=0.96)$. Two patients in the pre-intervention group and zero patients in the post-intervention group returned to a GHS clinic or the ED within 30 days for azithromycin-related adverse effects $(P=0.51)$. The two adverse effects experienced in the pre-intervention group included a patient-reported allergic reaction and disturbance in international normalized ratio (INR).

Inappropriate prescribing of azithromycin by attending physicians decreased by $7.0 \%$ from the pre- to post-intervention period $(85.1 \%$ vs. $78.1 \% ; P=0.06)$. Inappropriate prescribing by APPs decreased from 88.9 to $37.0 \%$ from the pre- to postintervention period $(P<0.001)$. Similarly, inappropriate prescribing by resident physicians decreased from the pre- to post-intervention period $(21.7 \% ; 66.7 \%$ vs. $45.0 \% ; P=$ $0.03)$. A higher percentage of inappropriate prescriptions originated from non-academic clinic sites in both the pre- and postintervention time period. The percentage of inappropriate prescriptions originating from non-academic clinics decreased by $11.2 \%(87.2 \%$ vs. $76.0 \% ; P=0.003)$.

\section{DISCUSSION}

Within the post-intervention period, there was a $12.6 \%$ reduction in inappropriate azithromycin prescriptions. Overall, the results of this study suggest that implementation of CDS panels influenced azithromycin prescribing. Although there was a reduction in unnecessary prescriptions post-intervention, the most common inappropriate indications for azithromycin were bronchitis or unspecified URI, suggesting that future stewardship targets should focus on improving prescribing for conditions that do not require antibiotics. Furthermore, this study demonstrated a decrease in inappropriate azithromycin prescribing within individual provider groups and within clinic practice settings after CDS intervention.

There are several possible explanations for the reduction in inappropriate azithromycin prescribing that was observed following CDS panel implementation. First, the CDS panel offered point-of-care syndrome-based guidance upon azithromycin ordering. Some information was available directly within the panel, such as reminders of inappropriate indications for azithromycin and alternative management for conditions that do not require antibiotics, whereas other information had to be actively sought by the provider via hyperlinks. Providing ordering options for alternative therapies based on indication directly within the panels may have impacted the improvement in inappropriate and unnecessary prescribing that was observed post-intervention. However, we are unable to measure how the providers utilized the information in the panels to influence therapeutic decisionmaking.

While this study demonstrated a statistically significant reduction in inappropriate azithromycin prescribing, the majority of azithromycin prescriptions remained inappropriate in the post-intervention period. Although this study was not designed to ascertain specific reasons for provider prescribing behavior, there are several potential factors that could be impacting the inappropriate azithromycin prescribing postintervention. One potential reason for inappropriate prescribing is concern for poor patient satisfaction scores. In a retrospective study of acute sinusitis, an indication to which antibiotics have low value, Sharp and colleagues observed that receipt of antibiotics was an independent predictor of favorable patient satisfaction scores. ${ }^{11}$ Ackerman and colleagues' survey found two highly perceived physician barriers regarding antibiotics prescribing for acute bronchitis: patient dissatisfaction if no receipt of antibiotics and patient expectations for antibiotics. ${ }^{12}$

This study demonstrated variability in prescribing among types of prescribers and prescribing sites. From the pre- to post-intervention period, there was a decrease in inappropriate prescribing within each provider group. However, there was a smaller percentage decrease in inappropriate azithromycin prescribing by attending physicians compared to resident physicians or APPs. In addition, this study also demonstrated a decrease in inappropriate prescribing from both academic and non-academic clinics in the post-intervention period, although the percentage decrease was smaller in the non-academic clinic setting. Although these results suggest that the CDS panel intervention appears to have broad applicability to different types of providers and practice sites, further studies are needed to evaluate the reasons for the variability in prescribing observed in this study.

There are several benefits associated with the intervention used in this study. Implementing CDS panels in the EMR offers a real-time intervention that impacts prescribing at the time of order entry. The intervention's incorporation into the EMR allows the CDS panels to have broad applicability to all providers, regardless of provider type or location of their clinical practice site. In addition, the CDS panels are a sustainable intervention that requires little maintenance other than updating recommendations when guidelines change. This provides opportunity for antibiotic stewardship programs to have an active role in outpatient antibiotic stewardship without devoting extensive personnel resources.

There are several limitations of this study that warrant consideration. The retrospective design of this study may have documentation biases leading to erroneous assignment of azithromycin prescriptions as either appropriate or inappropriate. For example, a patient prescribed azithromycin for a 
COPD exacerbation could have been considered inappropriate due to lack of documentation of signs and symptoms. Likewise, this study was limited in that there was a reliance on documented diagnosis rather than diagnostic criteria. The study being within a single healthcare system may limit the generalizability of these results to other institutions as response to such an intervention may differ. However, the intervention in this study was analyzed in various settings and provider types, suggesting it is an applicable intervention for various settings. This study only evaluated the impact of CDS panels on the use of azithromycin. Additional studies are required to determine if this intervention can impact the inappropriate prescribing of other antibiotics used for similar indications. Also, this study examined a short time frame of 6 months both pre- and post-intervention. It is unclear if benefits observed in post-intervention period are sustainable over longer periods. The periods examined differed between the two cohorts may have seasonal confounders such as the influenza season. The pre-intervention cohort encompassed months that were considered peak influenza season, where it is likely that more patients were presenting with URI symptoms and therefore creating a higher potential for inappropriate prescribing. Although there was no formal education on the CDS panels provided to clinicians as part of this study, medical directors could have provided education to providers on their own accord, and the quality or type of education was not measured. Future studies evaluating the use of CDS panels in conjunction with targeted provider education are needed. Finally, although this study did demonstrate a decrease in inappropriate prescriptions, click fatigue associated with the intervention was not able to be assessed.

In conclusion, our study suggests that implementation of a CDS order panel resulted in a reduction in inappropriate outpatient azithromycin prescribing. The CDS panel intervention appeared to have broad applicability to different types of providers and clinic sites. However, improvement is still needed in azithromycin prescribing for common outpatient conditions such as bronchitis or unspecified URI. Further studies into strategies for reducing prescribing variability and improvement in azithromycin prescribing are needed.

Acknowledgments: Howard Feldman for the technical development and feedback of the CDS order panels; Department of Pharmacy and Drug Information, Grady Health System
Corresponding Author: Alexandria May, PharmD, BCPS; Department of Pharmacy, Grady Health System, Atlanta, GA, USA (e-mail:avmay@gmh.edu).

\section{Compliance with Ethical Standards:}

This study was approved by the Emory University IRB, and for this type of study, formal consent was not required.

Conflict of Interest: The authors declare that they do not have a conflict of interest.

\section{REFERENCES}

1. Sanchez GV, Fleming-Dutra KE, Roberts RM, Hicks LA. Core elements of outpatient antibiotic stewardship. MMWR Recomm Rep 2016;65(No. RR-6): 1-12.

2. Fleming-Dutra KE, Hersh AL, Shapiro DJ, et al. Prevalence of inappropriate antibiotic prescriptions among US ambulatory care visits, 2010-2011. JAMA. 2016;315(17):1864-1873.

3. Tamma PD, Cosgrove SE. Addressing the appropriateness of outpatient antibiotic prescribing in the United States: an important first step. JAMA. 2016;315(17): 1839-41.

4. Shively NR, Buehrle DJ, Clancy CJ, Decker BK. Prevalence of inappropriate antibiotic prescribing in primary care clinics within a veterans affairs health care system. Antimicrob Agents Chemother 2018;62(8):e00337-18.

5. White AT, Clark CM, Sellick JA, Mergenhagen KA. Antibiotic stewardship targets in the outpatient setting. Am $\mathrm{J}$ Infect Control 2019;47(8):858-863

6. Gonzales R, Anderer T, McCulloch CE, et al. A cluster randomized trial of decision support strategies for reducing antibiotic use in acute bronchitis. JAMA Intern Med 2013;173(4):267-73.

7. Meeker D, Linder JA, Fox CR, et al. Effect of behavioral interventions on inappropriate antibiotic prescribing among primary care practices: a randomized clinical trial. JAMA. 2016:315(6):562-70.

8. Harris AM, Hicks LA, Gaseem A; High Value Care Task Force of the American College of Physicians and for the Centers for Disease Control and Prevention. Appropriate antibiotic use for acute respiratory tract infection in adults: advice for high-value care from the American college of physicians and the centers for disease control and prevention. Ann Intern Med 2016;164(6):425-434.

9. Chow AW, Benninger MS, Brook I, et al; Infectious Diseases Society of America. IDSA clinical practice guideline for acute bacterial rhinosinusitis in children and adults. Clin Infect Dis 2012;54(8): e72e112.

10. Centers for Disease Control and Prevention. Appropriate antibiotic use: community. Available at: https://www.cdc.gov/antibiotic-use/community/for-hcp/outpatient-hcp/adult-treatment-rec.html. Accessed June 26, 2020.

11. Sharp AL, Shen E, Kanter MH, Berman LJ, Gould MK. Low-value antibiotic prescribing and clinical factors influencing patient satisfaction. Am J Manag Care 2017;23(10):589-594.

12. Ackerman SL, Gonzales R, Stahl MS, Metlay JP. One size does not fit all: evaluating an intervention to reduce antibiotic prescribing for acute bronchitis. BMC Health Serv Res 2013;13:462.

Publisher's Note Springer Nature remains neutral with regard to jurisdictional claims in published maps and institutional affiliations. 